\title{
Pneumologische Erkrankungen und gastroösophagealer Reflux
}

\author{
Respiratory Diseases and Gastro-Esophageal Reflux
}

\section{P. Kardos}

Internistische Facharztpraxis, Zentrum f. Pneumologie, Allergie, Somnologie an der Klinik Maingau, Frankfurt am Main

VNR

2760512014144213351

Bibliografie

DOI http://dx.doi.org/

$10.1055 / \mathrm{s}-0034-1377880$

Pneumologie 2014; 68: 624-633

(c) Georg Thieme Verlag KC

Stuttgart · New York

ISSN 0934-8387

Korrespondenzadresse

Dr. med. Peter Kardos

Internistische Facharztpraxis

Zentrum f. Pneumologie,

Allergie, Somnologie an der

Klinik Maingau

Scheffelstraße 33

60318 Frankfurt am Main

info@Lungenpraxis-Maingau.de

\section{Lernziele}

Die gastroösophageale Refluxkrankheit (GERD) wird mit einer sich erweiternden Reihe von pneumologischen Erkrankungen in Verbindung gebracht, die mit oder ohne typische Refluxsymptome (Sodbrennen, Aufstoßen), postuliert als sog. stiller Reflux, in Erscheinung treten kann. Der vorliegende CME-Beitrag bewertet die aktuelle Literatur unter dem Gesichtspunkt der Assoziation und Kausalität und hilft dem Leser, vorhandene diagnostische Verfahren zu bewerten und diagnostische wie therapeutische Konsequenzen in der Patientenbetreuung zu erarbeiten.

\section{Einleitung}

Die gastroösophageale Refluxkrankheit (GERD) hat eine hohe Prävalenz in der Bevölkerung; in den USA ergab eine repräsentative Umfrage eine Prävalenz von $28 \%$ [1]. Eine Reihe von ebenfalls häufig vorkommenden, pneumologischen Erkrankungen (Asthma, chronischer Husten, idiopathische Lungenfibrose [IPF], chronisch obstruktive Lungenerkrankung [COPD], Bronchiektasen sowie Schlafapnoe) ist mit dem gastroösophagealen Reflux (GÖR) assoziiert. Der kausale Zusammenhang ist weniger klar. Dieser Beitrag beleuchtet die derzeitige Einschätzung der Rolle des GÖR bei pneumologischen Erkrankungen

\section{Definitionen \\ $\nabla$}

\section{Physiologischer Reflux}

Der Reflux von Mageninhalt in die Speiseröhre tritt unter physiologischen Bedingungen postprandial, vor allem tagsüber, aber auch spontan auf. Er ist die Folge spontaner Relaxationen des unteren Ösophagussphinkters (Transient Lower Esophageal Sphincter Relaxations, TLESR). 5-10\% der physiologischen spontanen Relaxationen des TLESRs sind schluckbedingt. Ein physiologischer GÖR wird nicht wahrgenommen. Ein pathologi- scher Reflux liegt dann vor, wenn die Exposition der Speiseröhre durch Refluat das physiologische Maß überschreitet.

\section{Messung des GÖRs}

Der Reflux wird mit der pH-Metrie im Ösophagus $5 \mathrm{~cm}$ oberhalb des unteren Ösophagussphinkters (Lower Esophagus Sphincter, LES) gemessen. Auch 2 oder 3 Kanäle (zusätzlich im Magen und im proximalen Ösophagus) können aufgezeichnet werden, im optimalen Falle in Ergänzung mit der Impedanzmessung (Multichannel Impedance Monitoring, MMI). Mit der 24h-pH-Metrie kann die saure Exposition ( $\mathrm{pH}-$ Wert <4) mit der Messung der ösophagealen Impedanz zusätzlich der schwach saure/alkalische Reflux gemessen werden. Wenn die kumulative Zeit mit pH-Werten $<4$ mehr als 4,5\% der 24h-Registrierzeit beträgt, liegt ein pathologischer (saurer) Reflux vor (95 Perzentile, d.h. 95\% aller Gesunden liegen bei oder unter diesem Wert) [2]. Eine genauere Beurteilung ermöglicht ein zusammengesetzter Score, DeMeester Score, der neben den Prozenten der Gesamtexpositionszeit 5 weitere klinisch wichtige Komponenten berücksichtigt und von der Software des pH-Messgeräts automatisch berechnet wird ( Tab. 1). Die obere Normgrenze liegt hier bei 14,72 [3].

Schwach saurer oder gar alkalischer Reflux („Volumenreflux“) kann mittels MMI erfasst werden. 6 Elektrodenpaare in $2 \mathrm{~cm}$ Abstand in der Sonde erlauben durch Änderung der Impedanz, Anzahl, Richtung (aboral nach oral oder umgekehrt) und Kinetik von jedem flüssigen Reflux neben dem pH-Wert zu erfassen. Der obere Normwert (95 Perzentile) liegt bei 73 Refluxepisoden in 24 Stunden. Nur ein Drittel der Refluxepisoden reicht bis zum oberen Ösophagus [4].

Durch eine Ösophagogastroskopie lässt sich eine Ösophagitis, die i.d.R. eine Folge von GÖR ist, zwar diagnostizieren. Es ist aber ein häufiges Missverständnis, dass bei negativem endoskopischem Befund GÖR ausgeschlossen werden kann: 
NERD (Non-erosive Reflux Disease) geht nicht mit durch eine Routine-Endoskopie erfassbaren Veränderungen einher.

Eine gastroösophageale Refluxerkrankung (GERD) liegt nach der Montreal-Definition dann vor, wenn der Reflux von Mageninhalt Beschwerden bzw. Symptome verursacht [5]. Gastroösophageal-typische Symptome sind Sodbrennen und Regurgitation (Aufstoßen), atypische gastroösophageale Symptome Dysphagia und Schmerzen ( Tab.2).

Extraösophageale Refluxsyndrome, auch extraösophageale Manifestationen (EÖM) genannt, die auch ohne „typische“ oder gar ganz ohne gastroösophageale Symptome auftreten können, werden in der 2006 veröffentlichten Montreal-Klassifikation [5] in 2 Kategorien eingeteilt: etablierte und vorgeschlagene Assoziationen.

Da ein Goldstandard in der klinischen Diagnose von GERD (und auch einiger assoziierten pneumologischen Erkrankungen wie z. B. Asthma) fehlt, können nur positive Therapiestudien eine Kausalität zwischen GERD und pneumologischen Erkrankungen beweisen.

Zur Frage der pulmonalen Manifestationen der GÖRD, die als EÖM angesehen werden, waren im Jahre 2006, zum Zeitpunkt der Erstellung der Montreal-Klassifikation, wenig schlüssige Literaturhinweise zu haben. Seither gibt es zumindest etwas mehr Licht in der Dunkelheit der möglichen Assoziationen und der Kausalität der obigen pneumologischen Erkrankungen; darüber hinaus sind COPD, Bronchiektasen und das obstruktive Schlafapnoesyndrom als weitere Assoziationen in Diskussion.

Es gibt keine Konsensus-Definition von Reflux mit extraösophagelen Symptomen. Der Terminus weist aber auf die Annahme hin, dass das Refluat den oberen Ösophagussphinkter überschreitet und Symptome im Bereich der oberen und unteren Atemwege hervorruft. Diese Annahme ist jedoch nicht unbedingt zutreffend.

In einer großen prospektiven in deutschsprachigen Ländern durchgeführten Kohortenstudie gaben 32,8\% von 6215 Patienten mit Sodbrennen und endoskopischer Untersuchung (die ProGERD Studie) EÖM an. Der Anteil von Patienten mit Sodbrennen und EÖM, aber ohne Ösophagitis war mit 30,5\% fast genauso hoch, wie bei Patienten mit Ösophagitis (34,9\%), wenngleich diese Differenz statistisch signifikant war [6]. Die Sensitivität und der negative prädiktive Wert der Endoskopie für GÖRD sind deshalb gering.

Der Pathomechanismus von EÖM ist nicht vollständig geklärt, es wird sowohl die Möglichkeit der lokalen Reizung (Mikroaspirationstheorie) [7] als auch der reflektorische Weg (Reflextheorie) diskutiert. Da aber keinesfalls jeder Refluxpatient mit oder ohne ösophageale („typische“) Refluxsymptomatik EÖM aufweist, wird zusätz-
Tab. 1 Komponente des DeMeester Scores.

\begin{tabular}{|ll|}
\hline $\begin{array}{l}\text { Zusammensetzung } \\
\text { DeMeester Score }\end{array}$ & Messeinheit \\
\hline Gesamtexpositionszeit & $\%$ \\
\hline Expositionszeit aufrecht & $\%$ \\
\hline Expositionszeit liegend & $\%$ \\
\hline Refluxepisoden & Anzahl \\
\hline lange Refluxepisoden (<5 Min) & Anzahl \\
\hline längste Refluxepisode & Minuten \\
\hline
\end{tabular}

Tab.2 Gastroösophageale Refluxkrankheit (GERD).

\begin{tabular}{|llll|} 
Gastroösophageale Symptome & \multicolumn{2}{l|}{ Extraösophageale Syndrome } \\
\hline Typisch & Atypisch & $\begin{array}{l}\text { Etablierte } \\
\text { Assoziationen }\end{array}$ & $\begin{array}{l}\text { Vorgeschlagene } \\
\text { Assoziationen }\end{array}$ \\
\hline Sodbrennen & Dysphagia & chronischer Husten & Pharyngitis \\
\hline Aufstoßen & Brustschmerzen & Laryngitis & Sinusitis \\
\hline & & Asthma & idiopathische Lungenfibrose \\
\hline & dentale Erosionen & Otitis media \\
\hline
\end{tabular}

lich eine entsprechende Prädisposition angenommen. Nur bei einer erniedrigten Reizempfindungsschwelle und pathologischer afferenter Funktion des Reflexes durch den Vagus mit Beeinflussung der Afferenz des unteren Ösophagussphinkters [8] wird eine EÖM, z.B. chronischer Husten ohne evidente Ursache [9-11], Asthma, Laryngitis etc. ausgelöst.

Andererseits handelt es sich bei den meisten EÖMs um multikausale Syndrome [12] , bei deren Diagnose deshalb auf die symptomassoziierte Wahrscheinlichkeit (Symptom Association Probability, SAP) zu achten ist $[8,13,14]$. So ist $z$. B. bei Impedanz pH-metrisch nur gering erhöhtem Reflux eine Kausalität mit chronischem Husten nur dann anzunehmen, wenn die überwiegende Mehrzahl der Hustenepisoden zeitlich mit einem Reflux assoziiert ist. Es ist üblich, die Assoziation in einem Zeitfenster von 2 Minuten zu betrachten.

Sodbrennen und Aufstoßen sind häufige Symptome und weisen auf GÖRD hin, die Prävalenz von Asthma und COPD ist hoch. Alleine dadurch ist eine Assoziation in der Epidemiologie pneumologischer Erkrankungen statistisch wahrscheinlich. Obwohl pathophysiologisch eine Kausalität in vielen Fällen „logisch“ wäre, steht deren evidenzbasierter Nachweis in den meisten Fällen noch aus.

\section{Reflux und Asthma}

Bereits vor mehr als 100 Jahren stellte Sir William Osler in seinem „The Principles and Practice of Medicine“ fest, dass Reflux Asthma auslöst [15]. Wenn ein Asthmapatient unter Anwendung adäquater verfügbarer Therapien keine Asthmakontrolle erreicht oder hierzu hochdosierte inhalative oder systemische Kortikosteroide in der

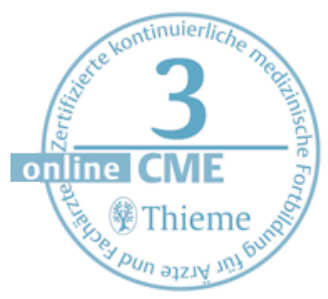


Tab.3 Longitudinale 3-Jahres-Kohortenstudien bei GERD (Studie 1) und Asthma (Studie 2) Patienten.

\begin{tabular}{|c|c|c|c|}
\hline $\begin{array}{l}1 \text { GERD Patienten } \\
n=5653\end{array}$ & $\begin{array}{l}\text { Vergleichskollektiv } \\
\mathrm{n}=\mathbf{8 1 0 5}\end{array}$ & $\begin{array}{l}2 \text { Asthmapatienten } \\
\mathrm{n}=9712\end{array}$ & $\begin{array}{l}\text { Vergleichskollektiv } \\
n=19334\end{array}$ \\
\hline Asthma entwickelt & Asthma entwickelt & GERD entwickelt & GERD entwickelt \\
\hline $\mathrm{n}=103$ & $n=99$ & $\mathrm{n}=219$ & $\mathrm{n}=241$ \\
\hline
\end{tabular}

Kombinationsbehandlung benötigt, handelt es sich nach der neuen ERS/ATS-Konsensus-Leitlinie [16] um ein schweres, therapieresistentes Asthma. Nach Überprüfung der Diagnose, der Inhalationstechnik und der Therapieadhärenz, nach Ausschluss anhaltender Exposition zu Allergenen oder Medikamenten, die Asthma verursachen können (z.B. Acetylsalicylsäure, non-steroidale Antiphlogistika, Betablocker), sollten Komorbiditäten und weitere Faktoren, die die Asthmakontrolle erschweren, evaluiert und behandelt werden. Hierzu gehört auch GERD mit typischen Refluxsymptomen. Die Leitlinie empfiehlt jedoch ausdrücklich, nicht ohne Vorhandensein typischer Refluxsymptome nach EÖM von Reflux zu suchen.

Die Asthma-Leitlinie der Deutschen Gesellschaft für Pneumologie und Beatmungsmedizin empfiehlt GERD (und chronische Rhinosinusitis) als Ursache für die verfehlte Asthmakontrolle zu prüfen $[17,18]$. Diese nationale und die internationale Empfehlung suggerieren, dass GÖR die Asthmakontrolle erschweren kann. Zumindest für erwachsene Asthmapatienten ist die Literatur diesbezüglich allerdings nicht eindeutig.

Der erste „Beweis“ für eine mögliche kausale Rolle von GÖR beim Asthma kommt aus der Epidemiologie: Assoziationen zwischen Asthma und Reflux wurden in mehreren älteren wie neueren Studien nachgewiesen [19-21]. Dabei ist die Prävalenz von Reflux bei Asthmapatienten zweifelsohne höher als in der Gesamtpopulation. Inwieweit aber die Prävalenz von Asthma bei Refluxpatienten erhöht ist, ist nicht eindeutig nachgewiesen [22]. Zur Illustration zeigt $\bullet$ Tab. 3 die Zahlen aus einer longitudinalen Inzidenzstudie [23].

In einer weiteren longitudinalen Kohortenstudie in 3 Ländern wurde das Neuauftreten von Asthma und nächtlichem GÖR (anamnestisch als Sodbrennen und/oder Aufstoßen im Liegen definiert) überprüft. Bei einem über die 9-jährige Beobachtungszeit persistierenden Reflux trat bei $13 \%$ der Teilnehmer signifikant häufiger Asthma neu in Erscheinung, gegenüber von $5,4 \%$, wenn von überhaupt keinem nächtlichen Reflux berichtet wurde [24]. Auch eine neu entstandene bronchiale Hyperreagibilität war häufiger, die adjustierte Odds Ratio war aber nicht signifikant. Die Lungenfunktion wies keine Veränderungen auf.

Betreffend solcher epidemiologischer Daten zum Reflux ist anzumerken, dass die meisten zitierten
Studien den Reflux anhand von Symptomen (Sodbrennen und Aufstoßen) oder in Registerstudien aufgrund der Einnahme von säurehemmenden Medikamenten diagnostizieren. Es ist (nicht nur) epidemiologisch ebenso schwierig Asthma zu diagnostizieren. Im Vergleich zur 24h-pH-Metrie ist die Sensitivität und Spezifität der verfügbaren Fragebögen unzureichend, selbst um typisch sauren Reflux zu diagnostizieren. So ist in einer Studie nur bei $72 \%$ der Asthmapatienten, die über Sodbrennen (GÖRD) geklagt hatten, pH-metrisch Reflux diagnostiziert worden. Andererseits hatten $29 \%$ der Asthmapatienten Reflux (GÖR), aber keine Refluxsymptome [25]. Noch problematischer ist die Diagnose von schwach saurem (Volumen-) Reflux.

Den zweiten „Beweis“ für die Assoziation zwischen Reflux und Asthma sollten therapeutische Studien liefern: Die Behandlung von Reflux sollte zur Besserung des Asthmas bzw. der Asthmakontrolle führen. Hier ist zwischen der medikamentösen Behandlung, die im Wesentlichen auf die Säurehemmung ausgerichtet ist, und der chirurgischen Behandlung zu unterscheiden, da letztere auch dem Volumen-Reflux entgegenwirkt.

Grundsätzlich ist festzustellen, dass die heute in erster Linie angewendete ProtonenpumpenHemmer (PPI) zwar die Säuresekretion, nicht jedoch den Reflux, nachweisbar mit der Impedanzmessung, hemmen. Allenfalls wird durch die PPI die Menge des Refluats geringfügig reduziert. Auch die Hemmung der Säuresekretion ist nicht komplett: Unter doppelter Standarddosis PPI ist die Speiseröhre nur zu 80\% der Zeit frei von Magensäure.

Mindestens 11 Therapiestudien zur PPI-Behandlung wurden publiziert, die größte im Jahr 2010 von Kiljander et al. Diese Studie war auch methodisch gut und schloss 961 Patienten unter ICS/ LABA-Kombinationstherapie mit unkontrolliertem Asthma ein. Sie hatten gleichzeitig GÖR, diagnostiziert als typische Refluxsymptome mind. 2-mal pro Woche während der 2-wöchigen Runin-Periode. Die 3-armige Studie untersuchte Plazebo, $40 \mathrm{mg}$ Esomeprazol und 2-mal $40 \mathrm{mg}$ Esomeprazol/Tag. Der morgendliche Peak Flow (PEF) war der primäre Zielparameter; beide Verumgruppen waren deutlich besser als Plazebo. Allerdings war der Unterschied gering: PEF 3,5 bzw. $5,5 \mathrm{ml} / \mathrm{min}$ gegenüber Plazebo. Ähnlich geringe, aber signifikante Unterschiede zeigten auch die FEV1 und die asthmabezogene Lebensqualität (AQLQ) - bei einer bemerkenswert großen Teilnehmerzahl. Allerdings liegt die statistische Besserung der Lebensqualität weit unter der MCID (Minimal Clinically Important Difference) und war damit für die betroffenen Patienten ebenso wenig wahrnehmbar wie die „Besserung“ der Lungenfunktionsparameter. Im Gegensatz hierzu haben sich die Refluxparameter in einem klinisch relevanten Ausmaß unter beiden Dosen von Esomeprazol gegenüber Plazebo gebessert [26]. 
Die Mitglieder des American Lung Association Asthma Clinical Research Centers haben eine multizentrische, nicht durch Pharmafirmen gesponserte Studie durchgeführt und 2009 im New England Journal of Medicine publiziert [27]. Sie haben ein anderes Herangehen gewählt. Unter der Annahme, dass unter mittelhoch oder hoch dosierter ICS-Therapie unkontrollierte Asthmapatienten mit klinisch manifesten Refluxsymptomen ohnehin einer PPI-Therapie bedürfen, wurden in diese 2-armige Studie (2-mal $40 \mathrm{mg}$ Esomeprazol vs. Plazebo) ausschließlich Patienten aufgenommen, die keine Refluxsymptomatik hatten, also ein EÖM (stiller Reflux) angenommen werden konnte. In Übereinstimmung mit den Daten der Literatur [25] hatten 40\% der 203 Verumund $41 \%$ der 199 Plazebo-Patienten einen pHmetrisch nachgewiesenen stillen Reflux. Der primäre Endpunkt war die Rate von Episoden mit Verschlechterung der Asthmakontrolle (PEF-Abfall $>30 \%$ an 2 konsekutiven Tagen, Notarztbesuch oder Notwendigkeit von OCS-Behandlung). Es konnte kein Unterschied zwischen Verum und Plazebo gezeigt werden. Wie in der Kiljander-Studie beschrieben, fand sich ein kleiner, klinisch irrelevanter, aber statistisch signifikanter Anstieg des morgendlichen PEF-Werts in der gleichen Größenordnung: $6 \mathrm{ml} / \mathrm{min}$.

Chan et al. führten 2011 eine Metaanalyse der beiden erwähnten und weiteren 7 bis dahin publizierten PPI-Therapiestudien bei Asthma durch [28]. Der primäre Endpunkt war die morgendliche PEF-Rate, die in 8 der 9 eingeschlossenen Studien ohnehin der primäre Endpunkt war. Die Metaanalyse zeigt einen kleinen, signifikanten Effekt der PPI-Therapie versus Plazebo von 8,68L/ min. Wenn die größte, die Kiljander-Studie, nicht berücksichtigt wurde, um den sogenannten Einzelne-große-Studie-Effekt auszuschalten, blieb die gleiche signifikante, aber klinisch nicht relevante PEF-Verbesserung. Wenn man nur die Subgruppe der 7 Studien analysiert, die nur Patienten mit Refluxsymptomen eingeschlossen haben (GÖR war pH-metrisch oder durch Refluxösophagitis gesichert worden), war die PEF-Verbesserung mit 16,9L/min etwas höher. Die MCID für die morgendliche PEF-Differenz liegt bei 15-20 L/min. Zur besseren Einschätzung der Ergebnisse: In vielen ICS/LABA-Asthma-Studien war eine durchschnittliche Besserung um über $30 \mathrm{~L} / \mathrm{min}$ gemessen worden.

Sekundäre Endpunkte wie abendlicher PEF, Lebensqualität und FEV1 zeigten in der Metaanalyse keine deutllichen Effekte gegenüber Placebo.
Auf der Basis der aktuellen Studienlage lässt sich die Kausalität zwischen Reflux und Asthma nicht beweisen. In den Therapiestudien wurde bislang nur ein klinisch irrelevanter Anstieg des morgendlichen PEFWertes konsistent nachgewiesen. Die diesbezügliche Empfehlung der neuen GINA-Leitlinie lautet:

- Bei unkontrolliertem Asthma ohne Refluxbeschwerden (Sodbrennen, saures Aufstoßen, Thoraxschmerz) ist ein GERD-Screening nicht erforderlich. - Bei Patienten mit Asthma und typischen Refluxbeschwerden ist eine empirische Refluxtherapie mit PPI zu erwägen, bei fortbestehenden Beschwerden eine weiterführende Diagnostik (24 h-pH-Messung, Endoskopie).

\section{Reflux und Husten}

Husten ist eines der häufigsten Symptome weshalb Patienten einen Arzt aufsuchen. Die meisten dieser Patienten leiden unter akutem Husten im Rahmen eines spontan abklingenden banalen viralen Infekts der oberen und/oder der unteren Atemwege, der in einzelnen Fällen bis zu 8 Wochen dauern kann. Chronischer Husten wurde daher als Husten, über 8 Wochen anhaltend, definiert. Die Ursache des chronischen Hustens kann durch Anamnese, klinische Untersuchung, Röntgenaufnahme (Lungentumoren, -parenchymerkrankungen) und Lungenfunktionsprüfung (COPD, Asthma) in den meisten Fällen geklärt werden, es kommt aber auch vor, dass all diese Untersuchungen negativ ausfallen und die Ursache des chronischen Hustens nicht evident wird [10]. In solchen Fällen wurde die Ursache des Hustens entlang der anatomischen Lokalisation der Hustenrezeptoren gesucht (sogenanntes anatomischdiagnostisches Protokoll [29]), Hustenrezeptoren finden sich am zahlreichsten im Bereich der oberen Atemwege; außerdem Trachea, Bronchien und auch in der Speiseröhre. So kann Reflux in die Speiseröhre, aber auch extraösophagealer, laryngo-pharyngealer Reflux Husten und andere EÖM experimentell auslösen [30,31].

Wenn die Ursache des chronischen Hustens nach Anamnese, klinischer Untersuchung, Röntgenaufnahme der Brustorgane und Lungenfunktionsprüfung unklar bleibt, könnte GÖR den Husten verursachen.

Die pathophysiologische Rationale diskutiert 2 mögliche Pathomechanismen für den Reflux bedingten Husten:

a) Reflextheorie: Der Husten wird auf reflektorischem Wege ausgelöst, der Reflexbogen läuft über sensible Vagusfasern zum Hustenzentrum. Der Husten wird durch einen gastro-ösophagealen Reflux in den distalen Ösophagus ausgelöst. Die Aktivierung des Hustenreflexes durch Reflux erzeugt einen Circulus vitiosus, da die Erhöhung des transdiaphragmalen Drucks beim Hustenstoß zur Öffnung des unteren Ösophagussphinkters führt und damit weiterer Reflux begünstigt wird.

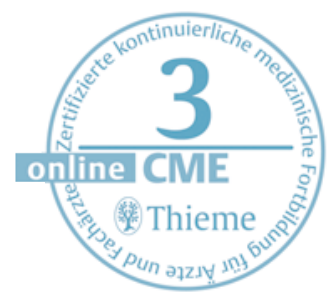


Tab.4 Messmethoden für Reflux-bedingten Husten.

\begin{tabular}{|c|c|c|c|c|}
\hline & $\begin{array}{l}\text { Diagnostische } \\
\text { Methode }\end{array}$ & Spezifität & Sensitivität & Anmerkung \\
\hline \multirow[t]{4}{*}{ Husten } & Fragebogen & $\downarrow$ & $\downarrow$ & Epidemiologie \\
\hline & VAS & $\downarrow$ & $\downarrow$ & \\
\hline & QOL & $\downarrow$ & $\downarrow$ & \\
\hline & Rekorder & $\uparrow$ & $\uparrow \uparrow$ & sehr aufwendig \\
\hline \multirow[t]{5}{*}{ Reflux } & Fragebogen & $\downarrow$ & $\downarrow$ & Epidemiologie \\
\hline & QOL & $\downarrow$ & $\uparrow$ & \\
\hline & pH-Metrie & $\uparrow \uparrow$ & $\uparrow$ & aufwendig \\
\hline & Impedanz pH-Metrie & $\uparrow \uparrow$ & $\uparrow \uparrow$ & niedrige Akzeptanz \\
\hline & Restech pH-Metrie & $\begin{array}{l}\text { noch nicht } \\
\text { bekannt }\end{array}$ & $\begin{array}{l}\text { noch nicht } \\
\text { bekannt }\end{array}$ & nur für LPR \\
\hline
\end{tabular}

Erklärung: $\downarrow$ niedrig; $\uparrow$ akzeptabel; $\uparrow \uparrow$ hoch

LPR: laryngo-pharyngealer Reflux

pH-Metrie: Messung des pH-Wertes in der Speiseröhre über 24 Stunden

Impedanz pH-Metrie: Messung aller Refluxereignisse (sauer und schwach sauer), wie weit proximal in der Speiseröhre sie reichen und des $\mathrm{pH}$-Werts

Restech pH-Metrie: Messung von aerosolisierter Säure im Rachen

b) Aspirationstheorie: Eine Regurgitation von Mageninhalt in flüssiger oder aerosolisierter Form zum proximalen Ösophagus und weiter bis zum Pharynx führt einerseits zu einer Schleimhautreizung im Bereich der oberen Atemwege (LPR, Laryngo-pharyngealer Reflux) und löst über die dort zahlreich vorhandenen Hustenrezeptoren den Husten aus. Andererseits kann das Refluat, ggf. nur in aerosolisierter Form, über Aspiration in die tiefere Atemwege gelangen und Husten auslösen. Dabei spielt potenziell nicht nur die Säure, sondern der leicht saure oder alkalische Volumenreflux mit den darin enthaltenen aggressiven proteolytischen Enzymen (Pepsin) eine Rolle. Dementsprechend können in vielen Fällen beim chronischen Husten mit Reflux im Speichel Pepsin, in der bronchoalveolaren Lavage Makrophagen nachgewiesen werden, welche aus dem Refluat stammende Lipide speichern. Ein solcher nicht saurer Reflux ist nur mit der kombinierten Impedanzmessung/pH-Metrie nachweisbar [13]. Es ist offensichtlich, falls ein solcher Pathomechanismus verantwortlich ist, können PPI therapeutisch wenig ausrichten.

Der Bernstein-Test ist für die breite klinische Diagnostik wegen seiner Invasivität und Untersuchungsdauer ungeeignet. In der Klinik erweist sich die Diagnose des Reflux-bedingten Hustens als sehr schwierig. Über die Treffsicherheit der in epidemiologischen Untersuchungen verwendeten Symptome für einen $\mathrm{pH}$-metrisch nachgewiesenen, pathologischen Reflux wurde bereits im Asthmakapitel berichtet. Tab. 4 fasst die Schwierigkeiten der Diagnostik zusammen.

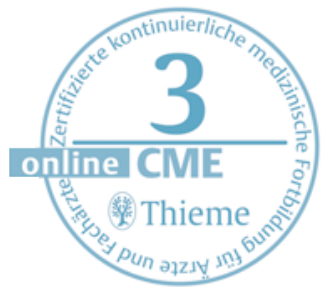

Bei fehlenden verlässlichen und breit anwendbarer Methoden, dem Goldstandard, in der Diagnostik des Hustens muss ein eventuell gegebener kausaler Zusammenhang in Therapiestudien nachgewiesen werden.

Stellvertretend für 19 einzelne Studien mit insgesamt 985 randomisierten Teilnehmern werden die Ergebnisse einer Cochrane Metaanalyse aus dem Jahre 2011 dargestellt [32]. Die federführende Autorin dieser Metaanalyse ist eine australische Kinderpneumologin. Sowohl 6 pädiatrische als auch 13 Erwachsenenstudien wurden eingeschlossen; 17 Studien verglichen PPI mit Plazebo, eine Studie Ranitidin mit PPI und eine Studie Cisaprid und Diät. In 18 der 19 Studien wurde der Reflux pH-metrisch bestätigt. Ein Teil der Studien wurde in HNO-Kliniken, der Rest in der Inneren Medizin durchgeführt. Der primäre Endpunkt, der Vergleich zwischen PPI und Plazebo fiel grenzwertig unter der Signifikanzschwelle ( $p=0,051)$ zugunsten des PPI-Armes aus, die Besserung des Hustens, gemessen mit dem Hustenscore in den Verum-Gruppen (Vergleich Studienbeginn/Studienende), war signifikant bei den Erwachsenen. Bei den Kindern zeigten sich keine signifikanten PPI-Effekte. Mehr Nebenwirkungen waren in den Verum-Armen aufgetreten. Hervorzuheben ist ein leicht erhöhtes Risiko für Pneumonie durch die Hemmung der Magensäure; bei Kindern ist dieses Risiko höher. Die Autoren schlussfolgern, dass angesichts der Schwierigkeit, schlechten Akzeptanz und Verfügbarkeit der pHMetrie bei Erwachsenen ein PPI-Therapieversuch gerechtfertigt sei; die Dauer sollte 2-8 Wochen betragen. Im Gegensatz dazu sei bei Kindern eine Probetherapie nicht angebracht.

Seit Erscheinen der Metaanalyse wurde eine weitere, monozentrische randomisierte Plazebo kontrollierte Studie mit Esomeprazol bei Erwachsenen publiziert [33]. Es ist bemerkenswert, dass die Ergebnisse (signifikante Besserung des Hustenscores vor/nach Behandlung, aber keine Besserung gegenüber Plazebo) mit der Metaanalyse identisch sind.

Schwach saurer (alkalischer) Reflux kann wirksam nur durch Antireflux-Chirurgie (Fundoplicatio) verhindert werden. Es gibt keine kontrollierte Studien über die Wirksamkeit operativer Methoden; eine retrospektive Studie [34] wertete $47 \mathrm{~Pa}$ tienten aus einem einzelnen Zentrum mit therapieresistentem Reflux-bedingtem Husten aus; alle wurden mit chirurgischer Fundoplicatio behandelt; 64\% der Patienten haben angesprochen. Die schwache Wirksamkeit der PPI-Therapie selbst bei pH-metrisch nachgewiesenem saurem Reflux-bedingtem Husten und die Tatsache, dass viele Patienten an saurem Reflux leiden, aber nur wenige hiervon chronischen Husten bekommen, lassen nach alternativen Erklärungen zu der Aspiration- und Reflextheorie suchen.

Smith et al. konnten durch sehr aufwendige Diagnostik (Spirometrie, Metacholin Provokationstes- 
tung, HR-CT und HNO-Untersuchung, Sputumuntersuchung auf Eosinophile, Zitronensäure-Hustenprovokation, Endoskopie und Manometrie des Ösophagus, Impedanz-pH-Metrie und objektive Registrierung des Hustens) bei 78 Patienten mit chronischem Husten nachweisen, dass die Hälfte der Hustenepisoden auf eine Refluxepisode folgte (47,9\%), und fast die Hälfte (43,8\%) hatte Husten mit nachfolgendem Reflux, ein Drittel der Patienten $(32,4 \%)$ hatte beides. Es stellte sich heraus, dass die Patienten, die auf Reflux husteten, nicht etwa einen stärkeren, länger dauernden Reflux oder Ösophagitis haben; sie unterschieden sich aber bei den Hustencharakteristika: Sie haben grundsätzlich mehr gehustet, und ihr Hustenreflex war auf Zitronensäure sensitiver. Im Gegensatz dazu: Die Patienten, die auf Husten Reflux bekamen, hatten grundsätzlich stärkeren Reflux, eher eine Refluxösophagitis und häufiger auch proximalen Reflux [8].

Wenn man Patienten mit chronischem Husten ohne evidente Ursache untersucht, bleiben ungeachtet ausführlicher Diagnostik etwa 20\% der Fälle ungeklärt: Man findet weder Reflux noch eine andere Ursache für den Husten [35]. Man spricht von chronischem idiopathischem Husten.

Diese Ergebnisse führten zu einer Modifikation der Aspirations- und Reflextheorie. Dem chronischen Husten liegt demnach eine (mit Zitronensäure- oder Capsaicin-Provokation objektiv messbare) Erhöhung der Sensitivität des Hustenreflexes zu Grunde. Dabei wird Husten selbst auf schwache Trigger wie Temperaturwechsel oder auch geringen, ggf. auch postprandial physiologischer Reflux ausgelöst [36]. Der afferente Bogen des Hustenreflexes ebenso wie die Afferenz für die Relaxation des unteren Ösophagussphinkters, die zu Reflux führt, verlaufen nebeneinander im Vagus. Das Hustenzentrum und die Regulierung des unteren Ösophagussphinkters sind im Hirnstamm ebenfalls benachbart und eine gemeinsame Einflussnahme wird angenommen ( $\bullet$ Abb.1).

Patienten mit typischen GÖR-Symptomen, die auch chronischen Husten haben, sollten probatorisch über 8 Wochen mit PPI behandelt werden. Patienten mit Verdacht auf ausschließlich EÖM (Husten) von Reflux profitieren nicht immer, ein Therapieversuch über 8 Wochen ist auch hier angezeigt. Bei fehlendem Ansprechen ist spezialisierte Refluxdiagnostik einschließlich Impedanz pH-Metrie erforderlich. Kinder unter 18 Jahren mit chronischem Husten sollten ohne Therapieversuch zur ausführlichen Refluxdiagnostik überwiesen werden.

\section{Reflux und idiopathische Lungenfibrose (IPF)}

Die Montreal-Klassifikation aus dem Jahr 2006

(৫ Tab.2) führt die IPF als mögliche, noch nicht etablierte Assoziation auf. Der heutige Stand der Literatur stützt zumindest die Kategorie der „etablierten Assoziation“.

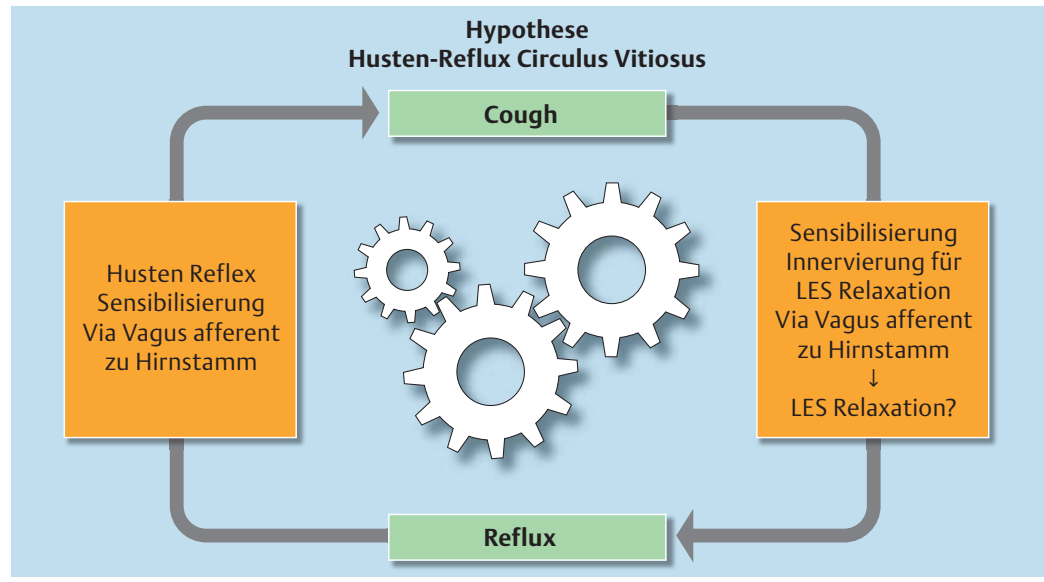

Abb.1 Die neue Hypothese des Reflux-bedingten Hustens.

Die IPF ist eine seltene Erkrankung, aber unter den idiopathischen interstitiellen Pneumonien die häufigste Entität. Der fibrotische Umbau des Lungenparenchyms führt zu einer steifen Lunge mit erniedrigter Vitalkapazität und Compliance. Letzteres bedeutet, dass größere transbronchiale Druckunterschiede erforderlich sind, um die Lunge in der Inspiration zu erweitern. Dies geht auch einher mit einer Zunahme des transdiaphragmalen Drucks durch die Notwendigkeit, höhere Negativdrucke im Thorax beim Einatmen aufzubauen. Es wird hypothetisch angenommen, dass die stark negative thorakale Druckschwankung den Übertritt von Mageninhalt in die Speiseröhre begünstigt, also den Reflux. Mikroaspiration von Mageninhalt (s. Kapitel Reflux und Husten) könnte als auslösender oder verschlimmernder Faktor für die Entstehung der Fibrose (mit)verantwortlich sein $[37,38]$. Die meisten diesen Patienten haben nur EÖM, also keine typischen Refluxsymptome [39].

Besonders ausgeprägt dürfte dieser Prozess in der IPF ähnlichen interstitiellen Erkrankung bei systemischer Sklerose sein, wo neben den Druckschwankungen auch eine Motilitätsstörung der Speiseröhre mit ausgeprägter Refluxneigung vorliegt [40].

Tatsächlich konnte in einer großen retrospektiven Studie [41] eine überzeugende Wirksamkeit von PPI in Hinblick auf die Progression der IPF nachgewiesen werden. Prospektive Studien laufen.

Pathophysiologische Überlegungen, die Messung von proximalen Reflux und mehrere - darunter eine mittelgroße retrospektive - Studien sprechen für eine kausale Rolle von Reflux bei IPF als EÖM. Die meisten Patienten haben keine typischen Refluxsymptome. Eine PPI-Therapie bei IPF-Patienten ist daher zu erwägen.

Cave: Interaktion von Pirfenidon (das einzige derzeit zugelassene Medikament für IPF) mit PPIs (CytochromP-Inducer, senkt den Pirfenidon Spiegel).

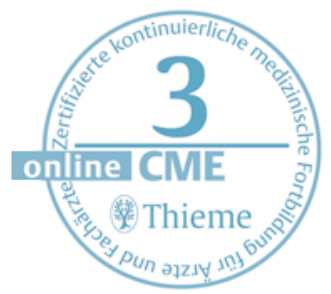




\section{Zusammenfassung}

Die Prävalenz von GÖR ist so hoch, dass Assoziationen mit anderen häufigen Erkrankungen wahrscheinlich sind. Der Nachweis der Kausalität ist schwierig, da Definition, Diagnostik und Messung von GÖR und insbesondere von stillem Reflux schwierig ist. Dies gilt auch für die meisten als etabliert geltende oder frisch auftauchende EÖM.

"Logische“ pathophysiologische Erklärungen, wie die Aspirationstheorie, sind nur Arbeitshypothesen. Sie müssen durch quantitative Untersuchungsergebnisse unterstützt und bei Fehlen eines diagnostischen Goldstandards durch entsprechende therapeutische Studien bestätigt werden.
Die meiste Literatur liegt für Reflux und Asthma bzw. Reflux und Husten vor, die Beweise für eine Kausalität sind lückenhaft. Die Effektivität der Refluxtherapie bei diesen Erkrankungen ist nicht überzeugend. Dies gilt für den stillen Reflux, d.h. die EÖM ohne typische Refluxsymptome.

Die Kausalität von GÖR bei IPF erscheint wahrscheinlich, die bisher vorliegenden Ergebnisse sind überzeugend, wenngleich nur eine kleine Anzahl der Patienten pH-metrisch ohne und mit PPI-Therapie gemessen wurde. Die Ergebnisse der multizentrischen laufenden Therapiestudien müssen abgewartet werden.

\section{Reflux und COPD}

COPD ist in der Montreal-Klassifikation ( $\bullet$ Tab.2) überhaupt nicht aufgeführt; in den letzten Jahren sind jedoch zahlreiche Publikationen zu diesem Thema erschienen. Sie weisen darauf hin, dass GÖR als symptomatischer, aber auch als stiller Reflux eine weitere EÖM sein könnte. GÖR ist mit COPD etwa genauso häufig assoziiert, wie mit Asthma [42], in dieser Studie in 37\% der pH-metrisch untersuchten COPD-Patienten. Ein Fünftel hatte keine typischen Refluxsymptome, also COPD nur als hypothetische EÖM. In einer anderen Studie hatten COPD-Patienten deutlich häufiger typische Refluxsymptome (25 vs. 9\%) [43], ein Déja vu aus der Asthma-Reflux-Epidemiologie. Es gibt auch dafür Hinweise, dass Reflux ein unabhängiger Risikofaktor für COPD-Exazerbationen ist [44].

Der gastroösophageale Reflux kommt auch bei Nicht-CF-Bronchiektasen (zystische Fibrose) überhäufig vor [42].

Für die Assoziation von Reflux und COPD gibt es pathophysiologische Hypothesen, epidemiologische Assoziationen, aber keine Therapiestudien.

\section{Reflux und Schlafapnoe (OSAS)}

Wie bei der IPF entstehen auch bei der obstruktiven Schlafapnoe durch den rezidivierenden Verschluss der oberen Atemwege hohe transdiaphragmale Druckschwankungen, die den Reflux begünstigen könnten, wenngleich dies eine sehr mechanistische Erklärung ist. Es konnte immerhin nachgewiesen werden, dass die CPAP-Therapie die Funktion des unteren Ösophagussphinkters verbessert und die Refluxepisoden eliminiert oder verringert [45]. Viele Patienten mit OSAS lei- den auch an Fettsucht, die sowohl Reflux als auch OSAS begünstigt. LPR ist häufig assoziiert mit Schnarchen, da Reflux von Mageninhalt in Larynx und Pharynx entzündliche Reaktionen und hierdurch sensorische Defizite hervorruft, die auch für die Obstruktion der oberen Atemwege verantwortlich sein könnten. Insofern ist eine strenge Assoziation nicht verwunderlich [24], eine Kausalität wurde bislang nicht nachgewiesen und erscheint eher unwahrscheinlich.

\section{Glossar: Abkürzungen}

EÖM Extraösophageale (Reflux) Manifestation

ERD Erosive reflux disease (Refluxösophagitis)

GÖR Gastroösophagealer Reflux

GERD Gastroösophageale Refluxkrankheit

LES Lower esophagus sphincter (unterer Ösophagus Sphincter

LPR Laryngo-pharyngealer Reflux

MII Multichannel intraluminal impedance

MMI pH-Metrie

Multichannel intraluminal impedance mit pH-Metrie

NERD Non-erosive reflux disease (mit Ösophagitis)

PPI Protonenpumpen Inhibitor

SAP Symptom association probability

\section{Interessenkonflikt}

$\nabla$

Der Autor erklärt, dass, das Thema dieser CMEFortbildung betreffend, kein Interessenkonflikt besteht.

\section{Literatur}

1 Camilleri $M$, Dubois D, Coulie B et al. Prevalence and Socioeconomic Impact of Upper Gastrointestinal Disorders in the United States: Results of the US Upper Gastrointestinal Study. Clin Gastroenterol Hepatol 2005; 3: $543-552$

2 Jamieson JR, Stein HJ, DeMeester TR et al. Ambulatory 24-h esophageal $\mathrm{pH}$ monitoring: normal values, optimal thresholds, specificity, sensitivity, and reproducibility. Am J Gastroenterol 1992; 87: 1102-1111

3 Johnson LF, DeMeester TR. Twenty-four-hour $\mathrm{pH}$ monitoring of the distal esophagus. A quantitative measure of gastroesophageal reflux. Am J Gastroenterol 1974; 62: $325-332$

4 Shay S. Esophageal impedance monitoring: the ups and downs of a new test. Am J Gastroenterol 2004; 99: $1020-1022$

5 Vakil N, van Zanten SV, Kahrilas P et al. The Montreal definition and classification of gastroesophageal reflux disease: a global evidence-based consensus. Am J Gastroenterol 2006; 101: 1900 - 1920

6 Jaspersen D, Kulig M, Labenz J et al. Prevalence of extraoesophageal manifestations in gastro-oesophageal reflux disease: an analysis based on the ProGERD Study. Aliment Pharmacol Ther 2003; 17: 1515-1520

7 Schreiber S, Garten D, Sudhoff $H$. Pathophysiological mechanisms of extraesophageal reflux in otolaryngeal disorders. Eur Arch Otorhinolaryngol 2009; 266: 17-24 
8 Smith JA, Decalmer S, Kelsall A et al. Acoustic cough-reflux associations in chronic cough: potential triggers and mechanisms. Gastroenterology 2010; 139: 754-762

9 Dinh QT, Heck S, Le DD et al. Pathophysiologie, Diagnostik und Therapie von chronischem Husten: Neuronale Reflexe und Antitussiva. Pneumologie 2013; 67: $327-$ 334

10 Kardos P, Berck H, Fuchs KH et al. Leitlinie der Deutschen Gesellschaft für Pneumologie und Beatmungsmedizin für Diagnostik und Therapie von erwachsenen Patienten mit akutem und chronischem Husten. Pneumologie 2010; 64: 336-373

11 Chung KF, McGarvey L, Mazzone SB. Chronic cough as a neuropathic disorder. The Lancet 2013; 1: 414-422

12 Katz PO, Gerson LB, Vela MF. Guidelines for the diagnosis and management of gastroesophageal reflux disease. Am J Gastroenterol 2013; 108: 308-328

13 Sifrim D, Dupont L, Blondeau $K$ et al. Weakly acidic reflux in patients with chronic unexplained cough during 24 hour pressure, $\mathrm{pH}$, and impedance monitoring. Gut 2005; 54: 449-454

14 Savarino $E$, Zentilin P, Inferrera $S$ et al. The relevance of symptom association analysis in GORD patients undergoing anti-reflux surgery. Gut 2012; 61: 326

15 Osler W. Bronchial Asthma. New York: Appleton; 1892: $497-501$

16 Chung KF, Wenzel SE, Brozek /L et al. International ERS/ATS guidelines on definition, evaluation and treatment of severe asthma. Eur Respir ] 2014; 43: 343 - 373

17 Buhl R, Berdel D, Criee CP et al. Leitlinie zur Diagnostik und Therapie von Patienten mit Asthma. Pneumologie 2006; 60: 139-177

18 Bel EH, Sousa A, Fleming L et al. Diagnosis and definition of severe refractory asthma: an international consensus statement from the Innovative Medicine Initiative (IMI). Thorax 2011; 66: 910-917

19 Gislason $T$, Janson C, Vermeire $P$ et al. Respiratory symptoms and nocturnal gastroesophageal reflux: a population-based study of young adults in three European countries. Chest 2002; 121: 158-163

20 Parsons JP, Mastronarde JG. Gastroesophageal reflux disease and asthma. Curr Opin Pulm Med 2010; 16: 60 - 63

21 McCallister JW, Parsons JP, Mastronarde JG. The relationship between gastroesophageal reflux and asthma: an update. Ther Adv Respir Dis 2011; 5: 143 - 150

22 Havemann BD, Henderson CA, El-Serag HB. The association between gastro-oesophageal reflux disease and asthma: a systematic review. Gut 2007; 56: 1654-1664

23 Ruigomez A, Rodriguez LA, Wallander MA et al. Gastroesophageal reflux disease and asthma: a longitudinal study in UK general practice. Chest 2005; 128: 85-93

24 Emilsson OI, Bengtsson A, Franklin KA et al. Nocturnal gastro-oesophageal reflux, asthma and symptoms of OSA: a longitudinal, general population study. Eur Respir J 2013; 41: $1347-1354$

25 Harding SM, Guzzo MR, Richter JE. 24-h esophageal pH testing in asthmatics: respiratory symptom correlation with esophageal acid events. Chest 1999; 115: 654659

26 Kiljander TO, Junghard O, Beckman $O$ et al. Effect of esomeprazole $40 \mathrm{mg}$ once or twice daily on asthma: a randomized, placebo-controlled study. Am J Respir Crit Care Med 2010; 181: $1042-1048$
27 The American Lung Association Asthma Clinical Research Centers. Efficacy of esomeprazole for treatment of poorly controlled asthma. N Engl J Med 2009; 360: 1487 1499

28 Chan WW, Chiou E, Obstein KL et al. The efficacy of proton pump inhibitors for the treatment of asthma in adults: a meta-analysis. Arch Intern Med 2011; 171: $620-629$

29 Irwin RS, Curley FJ. Is the anatomic, diagnostic work-up of chronic cough not all that it is hacked up to be? Chest 1989; 95: $711-713$

30 Bernstein L, Baker L. A clinical test for esophagitis. Gastroenterology 1958; 34: $760-781$

31 Ing AJ, Ngu MC, Breslin AB. Pathogenesis of chronic persistent cough associated with gastroesophageal reflux. Am J Respir Crit Care Med 1994; 149: 160-167

32 Chang AB, Lasserson TJ, Gaffney / et al. Gastro-oesophageal reflux treatment for prolonged non-specific cough in children and adults. Cochrane Database Syst Rev 2011: CD004823; DOI 10.1002/14651858.CD004823.pub4

33 Faruqi S, Molyneux ID, Fathi $\mathrm{H}$ et al. Chronic cough and esomeprazole: a double-blind placebo-controlled parallel study. Respirology 2011; 16: 1150-1156

34 Faruqi S, Sedman P, Jackson $W$ et al. Fundoplication in chronic intractable cough. Cough 2012; 8: 3

35 Kardos P, Gebhardt T. Chronisch persistierender Husten $(\mathrm{CPH})$ in der Praxis: Diagnostik und Therapie bei 329 Patienten in 2 Jahren. Pneumologie 1996; 50: 437 - 441

36 Morice $\mathrm{AH}$. Chronic cough hypersensitivity syndrome. Cough 2013; 9: 14

37 Wuyts WA, Agostini C, Antoniou KM et al. The pathogenesis of pulmonary fibrosis: a moving target. Eur Respir ] 2013; 41: 1207 - 1218

38 Savarino E, Carbone R, Marabotto E et al. Gastro-oesophageal reflux and gastric aspiration in idiopathic pulmonary fibrosis patients. Eur Respir ] 2013; 42: 1322-1331

39 Tobin R, Pope C, Pellegrini C et al. Increased prevalence of gastroesophageal reflux in patients with idiopathic pulmonary fibrosis. Am J Respir Crit Care Med 1998; 158: $1804-1808$

40 Savarino E, Bazzica M, Zentilin P et al. Gastroesophageal reflux and pulmonary fibrosis in scleroderma: a study using $\mathrm{pH}$-impedance monitoring. Am J Respir Crit Care Med 2009; 179: 408-413

41 Lee JS, Ryu JH, Elicker BM et al. Gastroesophageal reflux therapy is associated with longer survival in patients with idiopathic pulmonary fibrosis. Am J Respir Crit Care Med 2011; 184: 1390-1394

42 Lee AL, Button BM, Denehy L et al. Proximal and distal gastro-oesophageal reflux in chronic obstructive pulmonary disease and bronchiectasis. Respirology 2013; 19: 211 217

43 Phulpoto MA, Qayyum S, Rizvi N et al. Proportion of gastroesophageal reflux symptoms in patients with chronic obstructive pulmonary disease. J Pak Med Assoc 2005; 55: $276-279$

44 Liang B, Wang M, Yi Q et al. Association of gastroesophageal reflux disease risk with exacerbations of chronic obstructive pulmonary disease. Dis Esophagus 2013; 26: $557-560$

45 Shepherd K, Hillman D, Holloway $R$ et al. Mechanisms of nocturnal gastroesophageal reflux events in obstructive sleep apnea. Sleep Breath 2011; 15: 561 - 570

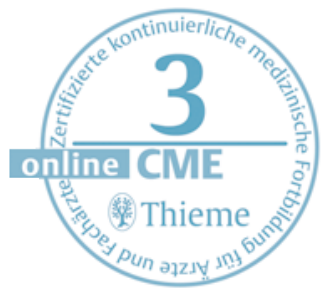




\section{CME-Fragen Pneumologische Erkrankungen und gastroösophagealer Reflux}

A Extraösophageale Manifestationen des gastroösophagealen Refluxes sind: nichtkardialer Thoraxschmerz, Dysphagie.

B Typische Refluxsymptome sind: Sodbrennen, Aufstoßen.

C Etablierte Manifestationen bei extraösophagealem Reflux sind: Asthma, chronischer Husten, chronische Laryngitis, dentale Erosionen.

D Zu den atypischen Refluxsymptomen gehören Dysphagie, Thoraxschmerz.

E Die Prävalenz typischer Refluxsymptome in der Allgemeinbevölkerung ist hoch.

Der Goldstandard der Diagnostik eines gastroösophagealen Refluxes ist

A ein validierter Fragebogen.

B die Endoskopie.

C ein validierter Fragebogen und Endoskopie.

D die 24h-pH-Metrie.

E die 24h-Impedanz pH-Metrie.

Ein nachgewiesener gastroösophagealer Reflux ist

A in jedem Falle pathologisch.

B nur dann pathologisch, wenn gleichzeitig eine Refluxösophagitis nachgewiesen wird.

C Ein pathologischer gaströosophagealer Reflux kann auch ohne Refluxösophagitis auftreten.

D Extraösophageale Manifestationen des Refluxes sind nur dann zu erwarten, wenn gleichzeitig der Reflux und die Ösophagitis nachgewiesen werden.

E Extraösophageale Manifestationen kommen nur in Verbindung mit Sodbrennen zustande.

\section{Asthma und Reflux: Welche Aussage trifft zu?}

A Asthmapatienten haben nicht häufiger einen Reflux als Gesunde.

B Durch die Infusion von 0,1N Salzsäure in den distalen Ösophagus (Bernstein-Test) kann ein reflektorischer Bronchospasmus ausgelöst werden.

C Der Bronchospasmus beim Bernstein-Test wird durch die unvermeidbare Aspiration ausgelöst.

D Durch die Protonenpumpenhemmer-Therapie kann bei nachgewiesenem gastroösophagealem Reflux die Asthmakontrolle und die Lebensqualität verbessert werden.

E Durch eine Protonenpumpenhemmer-Therapie kann nur die FEV1 verbessert werden.

\section{5}

Asthma und Reflux: Welche Aussage ist richtig?

A Die neue Asthma-Leitlinie GINA 2014 empfiehlt auf Therapiestufe 4 nicht kontrolliertes Asthma empirisch-probatorisch mit Protonenpumpenhemmer zu behandeln.

B GINA 2014 empfiehlt die empirische Protonenpumpenhemmer-Behandlung nur, wenn typische Refluxsymptome vorliegen.

C GINA 2014 empfiehlt die empirische Behandlung auch dann, wenn ausschließlich extraösophageale Manifestationen des Refluxes vorliegen.

D Die Ergebnisse der Protonenpumpenhemmer-Therapiestudien bei Asthma sind deshalb vorwiegend negativ, weil der Reflux vor dem Einschluss nicht pH-metrisch gesichert wurde.

E Die Wirkung der Protonenpumpenhemmer-Therapie auf die Asthmakontrolle wurde nur in wenigen, eher kleineren randomisierten kontrollierten Therapiestudien untersucht.

\section{Welche Aussage zum Refluat ist richtig?}

A Eine hochdosierte Protonenpumpenhemmer-Therapie ist für jede Art von Reflux ausreichend wirksam.

B Das Refluat aus dem Magen hat in jedem Fall einen sauren $\mathrm{pH}$-Wert.

C Unter Volumenreflux versteht man, dass viel saures Refluat aus dem Magen in die Speiseröhre gelangt.

D Unter Volumenreflux versteht man Refluat mit schwach saurem/alkalischem pH-Wert.

E Der Begriff Volumenreflux bezieht sich ausschließlich auf die große Menge des Refluats.

7 Welche Aussage zum chronischen Husten ohne richtungsweisenden Röntgenbefund und Lungenfunktion trifft zu?

A Wenn der Husten über 4 Wochen anhält, wird er als chronischer Husten definiert.

B Wenn der Husten über 8 Wochen anhält, wird er als chronischer Husten definiert.

C Gastroösophagealer Reflux kann nur dann als Ursache eines chronischen Hustens angenommen werden, wenn auch typische Refluxsymptome bestehen.

D Gastroösophagealer Reflux triggert den chronischen Husten immer durch Mikroaspiration.

E Wenn der Husten auf hochdosierten Protonenpumpeninhibitor nicht anspricht, kann Reflux als Ursache ausgeschlossen werden. 
8 Was trifft beim chronischen Husten ohne richtungsweisenden Röntgenbefund und Lungenfunktion zu?

A Für diesen Husten ist in den meisten Fällen Reflux verantwortlich.

B Schon geringe, auch physiologische, z. B. postprandiale Refluxepisoden können den Husten triggern.

C Je stärker der Reflux ausgeprägt ist (längere Refluxepisoden, höhere Säureexposition im Ösophagus, mehr proximaler Reflux), umso wahrscheinlicher ist, dass der Reflux Husten auslöst.

D Patienten mit starkem Husten provozieren durch transdiaphragmale Druckschwankungen starken Reflux.

E Jeder Patient mit starkem Reflux hustet, da das Refluat in der Speiseröhre den Husten zwangsläufig reflektorisch auslöst, etwa ähnlich wie bei der Aspiration.

Welche Aussage zu Reflux und idiopathische Lungenfibrose ist richtig?

A Die Montreal-Klassifikation 2006 ordnet die idiopathische Lungenfibrose als etablierte Assoziation mit dem gastroösophagealen Reflux ein.

B Die Wirkung von Protonenpumpeninhibitoren auf den progressiven Abfall der forcierten Vitalkapazität konnte in randomisierten kontrollierten Studien nachgewiesen werden.

C Nur die Wirkung von Protonenpumpeninhibitoren auf die Exazerbationen der IPF konnte in randomisierten kontrollierten Studien nachgewiesen werden.

D Es liegen retrospektive Studien mit Protonenpumpeninhibitoren vor, die für eine Wirkung auf den Abfall der forcierten Vitalkapazität und der Frequenz der Exazerbationen sprechen.

E Protonenpumpeninhibitoren sind gut verträglich, Interaktionen mit für die IPF zugelassenem Medikament (Pirfenidon) sind nicht zu fürchten.
10

Reflux und obstruktives Schlafapnoesyndrom:

Welche Behauptung ist falsch?

A Reflux und OSA haben gemeinsame Risikofaktoren.

B Intrathorakale/transdiaphragmale Druckschwankungen bei obstruktiver Apnoe führen zu erhöhtem Reflux von Mageninhalt in die Speiseröhre.

C Intrathorakale/transdiaphragmale Druckschwankungen bei obstruktiver Apnoe beeinträchtigen den unteren Ösophagussphinkter.

D Nasal continuous airway pressure (N-cpap)-Therapie verbessert die Sphinkterfunktion, verringert oder normalisiert die Säureexpositionszeiten im Ösophagus.

E Es besteht ein kausaler Zusammenhang: Reflux verursacht Schlafapnoe. 